\title{
Diagnosis and Treatment for the Kidney Stone in One West Highland White Terrier
}

\author{
Chunhui XU, Yong TAO* \\ Jiangsu Agri-animal Husbandry Vocational College (JAC), Jiangsu Region, China
}

*Corresponding Author: Yong TAO, Ph.D., Professor, engaged in animal science research, Taizhou 225300, China.

\begin{abstract}
On January $7^{\text {th }}$, 2020, a 7-year-old female West Highland White Terrier suffered from vomiting, purulent urination and its water intake was less, so it was sent to the hospital for treatment. Through blood routine examination, blood biochemical examination, $X$-ray examination and clinical examination, we made a diagnosis. The results showed that white blood cells and neutrophils were significantly increased, and the values of creatinine, urea nitrogen and total protein were abnormal, indicating renal failure. Through $X$-ray examination, it was diagnosed as kidney stone. We carried out an operation treatment, took out the stone from the kidney, and then carried out infusion, anti-inflammatory, and other conditioning measures after the operation. One week after the operation, removed the suture and the operative site achieved healing by first intention.
\end{abstract}

Keyword: Western Highland White Terrier; kidney stone; operation treatment

\section{INTRODUCTION}

Urolithiasis refers to a kind of urinary organ disease due to that inorganic salt or organic salt crystals form coagulates in the urinary tract, that is, stones or large amounts of crystals stimulate the mucosa of the urinary tract and cause bleeding, inflammation and obstruction [1]. Urolithiasis is a common disease in dogs, which can be divided into renal pelvis stones, ureteral stones, bladder stones and urethral stones according to the location of stones. The main clinical symptoms are unfluent urination, difficult urination, dysuria and hematuria [2]. Kidney stones in dogs usually occur in old dogs, and the frequency of kidney stones in males is higher than that in females. The causes of the disease are very complex. The main causes are diet, exercise and genetic problems. If the dog eats the food with high protein content for a long time, lacks drinking water, and the mineral content is too high, it will increase the burden on the dogs kidney and weaken the working ability of the kidney, which will increase the probability of stone formation. If the amount of exercise in dogs is not enough, it may result in a large number of inorganic salt deposition, which increases the burden on the kidney of dogs, thus forming stones. The genetic reasons are various, which may be congenital kidney insufficiency or congenital slow metabolism of the body.
There are other reasons, possibly some chronic diseases. Because the early symptoms of kidney stones in dogs are not obvious, they are often ignored or misdiagnosed as other diseases and make the disease from becoming worse. For example, if the ureter is blocked and compressed into the kidney, it will cause renal pelvis hydrops and kidney necrosis, and then cause bacterial infection, if not treated in time, it will cause sepsis, and serious cases can lead to death [3]. The diagnosis and treatment process of a case of kidney stone in West Highland White Terrier is reported as follows.

\section{INCIDENCE}

The female West Highland White Terrier admitted to the pet hospital on January 7th, 2020, was 7 years old, weighed $4 \mathrm{~kg}$, had been sterilized and had undergone mastectomy for breast cancer. The owner of the dog described that the dog had hematuria and purulent urine and it had had a kidney stone before. This time, it seemed to be a recurrence, so the dog was taken to get an examination. It was found that, within two days, the dog had intermittent vomiting, and its urine had white pus-like substance, and the amount of drinking water of the dog became less and the amount of urine decreased. The dog ate cooked chicken before vomiting 


\section{Clinical Examination}

After examine the dog' $\mathrm{s}$ condition, it was found that the body temperature was $39.2^{\circ} \mathrm{C}$, the pulse was 80 beats/min, and the visible mucosas were normal. Its weight is light, and it has lost about $300 \mathrm{~g}$ in two days. Then we palpated the dog. During the palpation, we pulled the fur of its back and neck to observe the recovery speed so as to judge that the dog had slight dehydration. Then we pressed the thoracic cavity, abdomen and back of the sick dog, and found that the thoracic cavity of the sick dog was normal, but there was obvious pain reaction in the abdomen and back of the sick dog. By checking again, we determined that the pain point was located in the back. Combined with the consultation, we preliminarily judged that it was a kidney problem, which caused the dog to vomit, pus in the urine, and decreased drinking water.

\section{LABORATORY EXAMINATION}

Results of blood routine examination before operation (Table 1). The results of biochemical test shown in Table 2. The X-ray film of preoperative anterioposterior examination (Fig.1). The X-ray film of preoperative lateral examination (Fig. 2).

Table1. Blood routine examination results

\begin{tabular}{|l|l|l|l|}
\hline Measured value of items & Result & Reference value & Tips \\
\hline WBCx $10^{9} / \mathrm{L}$ & 21.6 & $6.0-17$ & $\uparrow$ \\
\hline Lym \% & 3 & $0.8-5.1$ & - \\
\hline Segneutr \% & 16.8 & $4.0-12.6$ & $\uparrow$ \\
\hline Mono \% & 0.8 & $0.0-1.8$ & - \\
\hline RBC x $10^{12} / \mathrm{L}$ & 6.88 & $5.50-8.50$ & - \\
\hline Hemoglobin (RBC)x $10^{12} / \mathrm{L}$ & 174 & $110-190$ & - \\
\hline Segneutr \% & 82 & $35-75$ & $\uparrow$ \\
\hline Bandneutr \% & 0 & $0-3$ & - \\
\hline Eos $\%$ & 1 & $2-12$ & $\downarrow$ \\
\hline Platelets $(\mathrm{P}) \mathrm{x} 10^{9} / \mathrm{L}$ & 254 & $300-700$ & $\downarrow$ \\
\hline Hematocrit $(\mathrm{HCT}) \mathrm{L} / \mathrm{L}$ & 0.51 & $0.24-0.45$ & - \\
\hline
\end{tabular}

Table2. Biochemical examination results

\begin{tabular}{|l|l|l|l|}
\hline Measured value of items & Result & Reference value & Tips \\
\hline creatinine & 14.6 & $0.5-1.8$ & $\uparrow$ \\
\hline Urea nitrogen & 120 & $7-27$ & $\uparrow$ \\
\hline Total protein & 8.4 & $5.2-8.2$ & $\uparrow$ \\
\hline
\end{tabular}

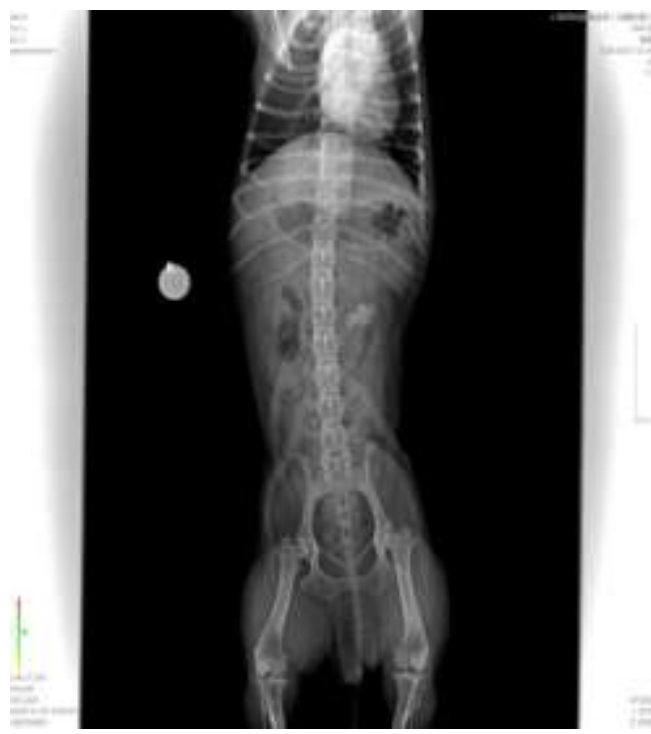

Fig. 1. X-ray film of preoperative anteroposterior examination

ARC Journal of Animal and Veterinary Sciences

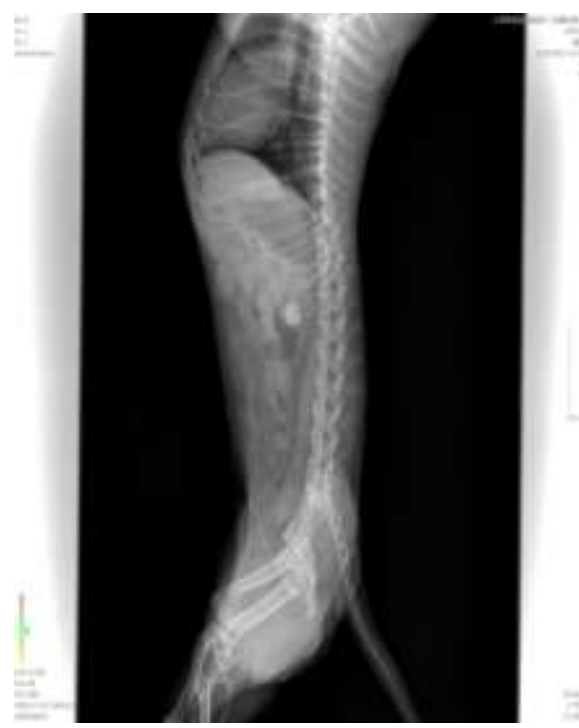

Fig. 2. X-ray film of preoperative lateral examination 


\section{DiAGNOSIS}

The results of hematology showed that the number of white blood cells was higher than the normal standard value. and the number of neutrophils significantly increased indicating that inflammatory reaction occurred in dogs; blood urea nitrogen, creatinine and blood phosphorus also exceeded the normal range, indicating the occurrence of azotemia and the disorder of phosphate metabolism function and that the kidney function of dogs was damaged; through X-ray, stone was found. In addition, the total protein content slightly increased, indicating that there is dehydration in the body. Based on the above results, the dog was diagnosed with kidney stones.

\section{Treatment}

\subsection{Preoperative Preparation}

Preparation for operation room. Check and disinfect surgical instruments. Check the air tightness of the ventilator, trachea and anesthesia machine, check the intubation model and the spare model and disinfect. Check whether the dosage of anesthetic drug called, isoflurane is sufficient to complete the operation. Prepare the laryngoscope, turn on the ECG monitoring equipment and check whether it works normally, and disinfect the operating table, heat it up, and adjust the height and radian of the operating table.

Preoperative medication for dogs. Atropine is used to assist anesthesia. The analgesic used is Meloxicam, and the hemostatic used is Hemocoagulase. Use subcutaneous injection method and embed the indwelling needle in the right forelimb. The dog was injected with needles before the operation, and 15 minutes later, the dog was brought into the operating room to undergo operation.

\subsection{Operative Treatment}

Before the operation, inject $0.6 \mathrm{~g} / \mathrm{kg}$ propofol intravenously to anesthetize the sick dog. When the dog fell into unconsciousness, we quickly inserted the tracheal cannula into it and then connected the ventilator, anesthesia machine and ECG monitoring equipment. Sterilize the integument and cut the skin from the abdomen midline to the peritoneum. We found the kidney and found that it was swollen and deformed, and the vessels in the kidney capsule proliferated, and then we used nephrolithotomy, and after careful examination, we first bound the renal artery and vein to control the amount of bleeding. Perform cortex tomy and medulla tomy. Make an incision on the back of the left kidney, cut the renal pelvis, and then take out the stones and blood clots. Check the ureter on the same side, and then insert the dog's special catheter into the ureter from the incision through the renal pelvis. Irrigate with warm sterilized normal saline until the fluid can enter the bladder end smoothly through the ureter, and then flush the residual blood clot. After examination, we found the cortex of the left kidney had become very thin. Therefore, we speculated that the renal function of the dog damaged. Suture the tubercle. In order to control the amount of bleeding in the kidney, we made retension suture across the kidney. Check the bladder, irrigate the residual stones with warm sterilized normal saline, and then rinse them. Use sterile gauze to absorb the hydrops left in the abdominal cavity during flushing. After checking to make sure there is no bleeding point, we sutured the skin. Flush the abdominal cavity and close it routinely. According to the suture speed, close the anesthesia machine as soon as possible. When the dog had spontaneous breathing, we removed the ventilator. Then we pull out the tracheal cannula and wait for the dog to wake up.

\subsection{Postoperative Care}

Carry out infusion therapy and give antibiotics to prevent postoperative infection and inflammation. There was hematuria for two consecutive days after the operation, and on the 3rd day, the urine was clear and the spirit of the dog was improved. After that, daily prescription food and continuous infusion, and no hematuria. Within 7 days after operation, the blood routine and blood biochemical indexes returned to the normal range. 9 days later, the dog was in a normal state of mind, then we carried out a biochemical examination of the blood, and the result showed that the azotemia was not so serious, and then removed the suture, and the operative site had healing by first intention.

\section{DISCUSSION}

The dog's lower urinary tract stone refers to the urinary tract inflammation caused by the coagulation of inorganic or organic crystal in the urinary tract, which can cause frequent urination (usually hematuria) or even blockage or both. It is a common clinical disease of urinary system [4]. Due to the mechanical stimulation of the bladder and urethra by stone, destroyed and damaged the integrity of bladder and urethral mucosa; if accompanied by bacterial infection, it will cause cystitis and urethritis, and even 
induce systemic symptoms. When the stones go down and block the urethra, the sick dogs will urinate urgently and frequently, or anuria will appear. If the dog not treated in time, bladder rupture or uremia may occur, threatening the life of the dog [5]. The epidemiological investigation of urinary tract stones in dogs showed that the incidence rate of this disease is $0.58 \% \sim 1.67 \%$ in China, and the recurrence rate is $8.0 \% \sim 14.58 \%$. The incidence rate of $\operatorname{dog}$ urolithiasis outside China is $0.05 \%$ to $1 \%$ [6]. It is clear that the incidence rate of dog urolithiasis in China is higher than that in other countries, which shows the clinical importance and regional characteristics of this disease. Urolithiasis in the lower urinary tract is more common, accounting for $95.98 \%$ of the total cases. Urolithiasis of the upper urinary tract is rare, accounting for $4.02 \%$ of the total cases. The incidence rate of male dogs is slightly higher than that of female dogs because of their different physiological and structural characteristics. Most of the urolithiasis of male dogs were found in the bladder and urethra, while the urolithiasis of female dogs were found in the bladder. The urethra of male dogs is longer than that of female dogs, and the curvature of urethra is larger, the thickness of urethra is uneven, and there are obvious narrow parts, and they can all cause stone discharge to be obstructed [7].

The possible reasons for the formation of kidney stone are the bacterial descending infection or ascending infection on the dog' s kidney, which causes the epithelial cells of the kidney to separate and form a core, and then calcium, phosphorus and magnesium ions begin to deposit to form stones. Kidney stones are uncommon in dogs and cats, with an incidence of about $4 \%$. Among the components of kidney stones, the incidence of calcium oxalate is $39 \%$, struvite is $35 \%$, uratoma is $8 \%$, calcium phosphate stone is $4 \%$, and of course, there are stones of other components [8].

Before operation, we need to assess whether the dogs have metabolic abnormalities caused by stones. We can use blood biochemical methods, such as serum creatinine and urea nitrogen, to evaluate renal function. We used X-ray film to determine the location and number of stones in the urinary tract to determine the location of obstruction, and we used antibiotics to control urinary tract infection.

After operation, we should keep on giving intravenous infusion to the dog to correct azotemia, and pay attention to whether the dog urinates. We can wear double channel catheters and bags for human use to determine urine output and maintain normal urine excretion. After the operation, we need to take X-ray film again to check if all stones are removed. Choose appropriate antibiotics and dosages to prevent kidney infections in dogs.

Adhering to a reasonable and correct diet system can reduce the recurrence rate of kidney stones in dogs to 60\% [9]. For preventing calcium stones, most of the methods emphasize eating less high calcium food, but insufficient calcium intake may cause the formation of calcium oxalate stone. Oxalic acid in urine is easier to increase the saturation of calcium oxalate than calcium, and the difference between them is ten times. Therefore, the intake of oxalic acid should be appropriate. Common foods, such as spinach, tea, strawberries, nuts, etc., should be eaten less. The intake of meat protein is not suitable for too much, which will affect the increase of urinary calcium excretion. The consumption of sodium chloride should also be appropriately limited, because too much sodium in urine will accelerate the formation of stones. For non calcium stones, low purine diet should be adopted to reduce uric acid stone, and the main forbidden foods are animal viscera and seafood, because this kind of food can reduce the concentration of uric acid in urine, but proper intake of this kind of food will help to control the growth and recurrence of cystine stone.

\section{ACKNOWLEDGMENT}

This work is supported by "National Development and Reform Commission of China's Bio-breeding Capacity Building Project" (20150299); "Jiangsu Modern Agricultural Industrial Technology System Construction Project" (JATS2018243).

\section{REFERENCE}

[1] Ling G V , Franti C E , Ruby A L , et al. Urolithiasis in dogs. I: Mineral prevalence and interrelations of mineral composition, age, and sex. American Journal of Veterinary Research, 1998, 59(5):624.

[2] Zhuo Guorong, Ding Xingxing. Etiological analysis of bladder stone and urethral stone in dogs and clinical surgical treatment experience. Heilongjiang Animal Science and Veterinary Medicine, 2007, 2007 (008): 86-87

[3] Jiang Jiajin, Tao Qingshu. Etiological analysis of canine urolithiasis and the clinical diagnosis and treatment. Journal of Jinling Institute of Technology, 2005, 21 (3): 84-86 
[4] Zhou Qiuping, Jin Yinji, Shi Yibing, etc. Examination and cause analysis of 56 cases of dog urinary stones $[\mathrm{J}]$. Animal Husbandry and Veterinary Medicine, 2003, 35 (4): 35-37

[5] Zhang Ningning, Liu Wei and Lin Degui. Investigation and analysis of the epidemic status of urolithiasis of dogs. Chinese Journal of Veterinary Medicine. 2006, 42(11):41-43.

[6] Xiang Fu, Tian Haiyan. One case of diagnosis and treatment of bladder and kidney stones in a $\operatorname{dog}[\mathrm{J}]$. China Animal Husbandry \& Veterinary Medicine, 2009, 36 (8): 3-5
[7] Sun Weidong, Wang Jinyong, Liu Yongwang, et al. Investigation of dog urolithiasis in Nanjing area and analysis of urinary stone composition [J]. Chinese Journal of Veterinary Medicine, 2007, 27 (6): 935-937,

[8] Dong Jun. Manual for pet disease diagnosis and treatment and formulary [M]. Beijing: Chemical Industry Press, 2012, 4:178-180

[9] Li Zengmin, Min Fenglong. One case of diagnosis and treatment of kidney stone complicated with bladder stone in a dog. Chinese Journal of Traditional Veterinary Science, 2012, (2): 42-43

Citation: Chunhui XU, Yong TAO. Diagnosis and Treatment for the Kidney Stone in One West Highland White Terrier. ARC Journal of Animal and Veterinary Sciences. 2020; 6(2):18-22. DOI:https://doi.org/10.20431/2455-2518.0602003.

Copyright: (C) 2020 Authors. This is an open-access article distributed under the terms of the Creative Commons Attribution License, which permits unrestricted use, distribution, and reproduction in any medium, provided the original author and source are credited. 